\title{
The kinematics of the quadrupolar nebula M 1-75 and the identification of its central star ${ }^{\star}$
}

\author{
M. Santander-García ${ }^{1,2,3}$, P. Rodríguez-Gil ${ }^{1,2,3}$, O. Hernandez ${ }^{4}$, R. L. M. Corradi2 ${ }^{2,3}$, D. Jones ${ }^{1,5}$, C. Giammanco ${ }^{2,3}$, \\ J. E. Beckman ${ }^{6,2,3}$, C. Carignan ${ }^{4}$, K. Fathi ${ }^{7}$, M. M. Rubio-Díez ${ }^{1,8}$, F. Jiménez-Luján ${ }^{1,9,10}$, and C. R. Benn ${ }^{1}$ \\ 1 Isaac Newton Group of Telescopes, Ap. de Correos 321, 38700 Sta. Cruz de la Palma, Spain \\ e-mail: msantander@ing.iac.es \\ 2 Instituto de Astrofísica de Canarias, 38200 La Laguna, Tenerife, Spain \\ 3 Departamento de Astrofísica, Universidad de La Laguna, 38205 La Laguna, Tenerife, Spain \\ ${ }^{4}$ LAE, Université de Montréal, CP 6128 Succ. Centre Ville, Montréal, QC H3C 3J7, Canada \\ Jodrell Bank Centre for Astrophysics, School of Physics and Astronomy, University of Manchester, Manchester, M13 9PL, UK \\ ${ }^{6}$ Consejo Superior de Investigaciones Científicas, Madrid, Spain \\ 7 Stockholm Observatory, Department of Astronomy, Stockholm University, AlbaNova, 10691 Stockholm, Sweden \\ 8 Centro de Astrobiología, CSIC-INTA, Ctra de Torrejón a Ajalvir km 4, 28850 Torrejón de Ardoz, Spain \\ 9 Instituto de Física de Cantabria (CSIC-Universidad de Cantabria), 39005 Santander, Cantabria, Spain \\ 10 Dpto. de Física Moderna, Universidad de Cantabria, Avda de los Castros s/n, 39005 Santander, Cantabria, Spain
}

Received 21 April 2010 / Accepted 2 June 2010

\section{ABSTRACT}

\begin{abstract}
Context. The link between how bipolar planetary nebulae are shaped and their central stars is still poorly understood. Aims. This paper investigates the kinematics and shaping of the multipolar nebula M 1-75, and briefly discusses the location and nature of its central star.

Methods. Fabry-Perot data from GH $\alpha$ FAS on the WHT that samples the Doppler shift of the [NII] $658.3 \mathrm{~nm}$ line are used to study the dynamics of the nebula by means of a detailed 3D spatio-kinematical model. Multi-wavelength images and spectra from the WFC and IDS on the INT, as well as from ACAM on the WHT, allowed us to constrain the parameters of the central star.

Results. The two pairs of lobes, angularly separated by $\sim 22^{\circ}$, were ejected simultaneously approx. $\sim 3500-5000$ years ago, at the adopted distance range from 3.5 to $5.0 \mathrm{kpc}$. The larger lobes show a slight degree of point symmetry. The formation of the nebula could be explained by wind interaction in a system consisting of a post-AGB star surrounded by a disc warped by radiative instabilities. This requires the system to be a close binary or a single star that engulfed a planet as it died. On the other hand, we present broadand narrow-band images and a low $\mathrm{S} / \mathrm{N}$ optical spectrum of the highly-reddened, previously unnoticed star that is likely the nebular progenitor. Its estimated $V-I$ colour allows us to derive a rough estimate of the parameters and nature of the central star.
\end{abstract}

Key words. planetary nebulae: general - planetary nebulae: individual: M 1-75 - planetary nebulae: individual: PN G068.8-00.0 ISM: kinematics and dynamics

\section{Introduction}

Planetary nebulae (PNe) represent the terminal breath of $90 \%$ of the stars in the Universe. However, their shaping mechanism is still poorly understood. Bipolar PNe present undoubtedly the most challenging case. Several attempts have been made to explain how they are shaped (see the review by Balick \& Frank 2002), which break spherical symmetry by invoking elements that fall in two distinct categories: a) rapid stellar rotation and/or magnetic fields (e.g. García-Segura et al. 1999; Blackman et al. 2001); and b) a close interacting companion to the star (e.g. Nordhaus \& Blackman 2006; for a review see de Marco 2009). The latter hypothesis seems to be gaining some

* Based on observations made with the $4.2 \mathrm{~m}$ William Herschel Telescope and the $2.5 \mathrm{~m}$ Isaac Newton Telescope, both operated on the island of la Palma by the Isaac Newton Group of Telescopes in the Spanish Observatorio del Roque de los Muchachos of the Instituto de Astrofísica de Canarias. ground as close binary systems are progressively being found (e.g. Miszalski et al. 2009; Miszalski et al., in prep.) at the cores of bipolar PNe.

Spatio-kinematical modelling of PNe constitutes an excellent tool for testing theoretical models. It provides us with important parameters to be matched by the different models of formation, such as the 3D morphologies and velocity fields of the outflows, their kinematical age (once disentangled from the distance to the nebula) and their orientation to the line of sight.

M 1-75 (PN G068.8-00.0, $\alpha=200444.086 \delta=+3127$ $24.42 \mathrm{~J} 2000$ ) is a good example of a complex nebula. It displays a seemingly irregular horseshoe-like central region, out of which two systems of faint lobes emerge. It was first classified as quadrupolar by Manchado et al. (1996b), and a tentative attempt to recover its kinematic parameters was made by Dobrinčić et al. (2008).

In this paper we present Fabry-Perot interferometry of M 1-75, from which we derive a detailed spatio-kinematical 


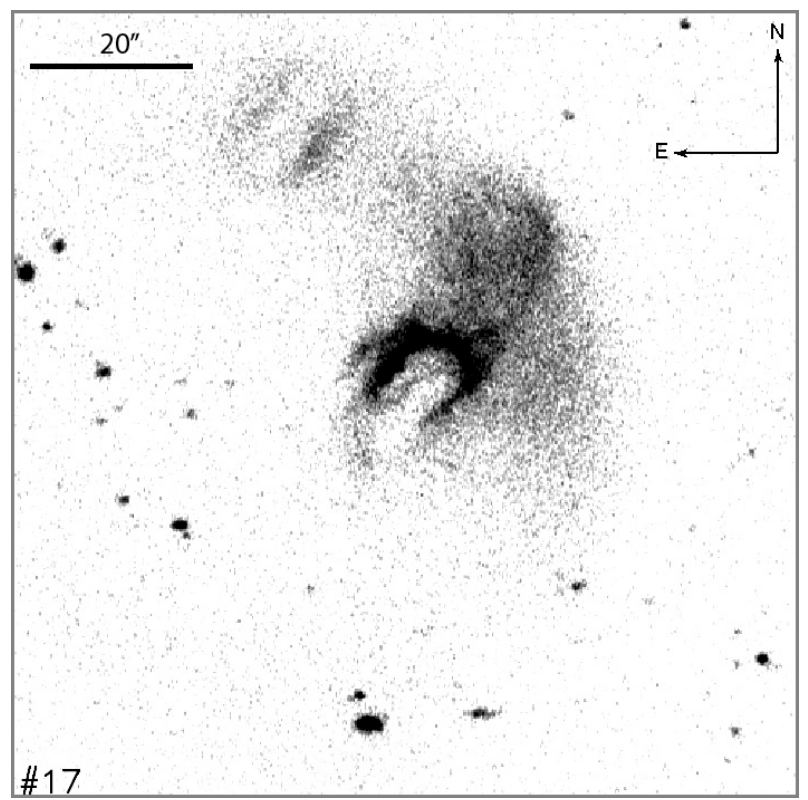

Fig. 1. Channel \#17 of the GHAFAS datacube, showing the blue-shifted upper side of the horseshoe (centre). The faint emission from the large lobe extending northwest is contaminated by a broad arc-shaped artifact which spans over several channels. A fainter version of the horseshoe itself is replicated as a ghost near the top left corner of the frame. Additionally, several channels are slightly contaminated by $\mathrm{H} \alpha$ emission from adjacent orders. These artifacts, however, do not prevent a proper modelling of the nebula (see Sect. 3).

model (Sect. 3). We also report the first imaging and spectroscopic detection of its central star (Sect. 4). We then discuss both results and their implications in the formation of the nebula (Sect. 5).

\section{Observations and data reduction}

\subsection{Fabry-Perot interferometric data}

The [NII] $658.3 \mathrm{~nm}$ emission of M 1-75 was scanned with GH $\alpha$ FAS (Galaxy H $\alpha$ Fabry-Perot System) on the $4.2 \mathrm{~m}$ WHT (William Herschel Telescope) on July 6, 2007, as part of its commissioning programme. The nebula was observed in highresolution mode with the OM4 etalon (resolving power $R \sim$ 18000 , effective finesse $\mathfrak{J}_{\mathrm{e}}=24$ ) and a plate scale of 0.2 pixel $^{-1}$. The free spectral range was $8.62 \AA$ or $392 \mathrm{~km} \mathrm{~s}^{-1}$ split into 48 channels, thus leading to a velocity step of $8.16 \mathrm{~km} \mathrm{~s}^{-1}$ per channel. The total exposure time of the scanning was $1.9 \mathrm{~h}$, and the seeing $0{ }^{\prime} 8$. The instrumental response function (IRF) was measured by fitting a Lorentzian to the profile of a Neon lamp line and resulted in an instrumental width (FWHM) of $18.6 \mathrm{~km} \mathrm{~s}^{-1}$.

The data were reduced following the standard procedure for GH $\alpha$ FAS data, which are described in Hernandez et al. (2008). Several artifacts persisted through the data reduction process. These include slight contamination by $\mathrm{H} \alpha$ emission from adjacent orders (specially in the first and last channels of the datacube), a ghost of the inner region of the nebula, and an arcshaped artifact which runs across several channels, at different locations (see Fig. 1).
Table 1. Log of the broad and narrow-band imaging observations.

\begin{tabular}{lccccc}
\hline \hline Date & $\begin{array}{c}\text { Telescope/ } \\
\text { Instrument }\end{array}$ & $\begin{array}{c}\text { Filter } \\
\text { ref. }\end{array}$ & Band & $\begin{array}{c}\text { Exp. time } \\
(\mathrm{s})\end{array}$ & Seeing \\
\hline 2009 Jun. 11 & WHT/ACAM & $\# 17$ & $I$ & $3 \times 120$ & $1^{\prime \prime \prime} 4$ \\
2009 Jun. 12 & INT/WFC & $\# 201$ & Str. Y & $2 \times 600$ & $1^{\prime \prime} 3$ \\
2009 Jun. 12 & INT/WFC & $\# 197$ & $\mathrm{H} \alpha$ & 120 & $1^{\prime \prime} 3$ \\
2009 Sep. 10 & INT/WFC & $\# 204$ & $U$ & 1200 & $1^{\prime \prime} 6$ \\
2009 Sep. 10 & INT/WFC & $\# 204$ & $B$ & 1200 & $1^{\prime \prime} 6$ \\
2009 Sep. 10 & INT/WFC & $\# 204$ & $V$ & 1800 & $1^{\prime \prime} 6$ \\
2009 Sep. 10 & INT/WFC & $\# 204$ & $I$ & 600 & $1^{\prime \prime} 6$ \\
2009 Sep. 10 & INT/WFC & $\# 204$ & Str. Y & 600 & $1^{\prime \prime} 6$ \\
\hline
\end{tabular}

\subsection{Broad and narrow-band imaging}

Several images of M 1-75 in the light of different filters $(U, B, V$, $I, \mathrm{H} \alpha$ and Strömgren Y) were taken with ACAM (Auxiliary-port Camera) on the WHT and with the WFC (Wide Field Camera) on the $2.5 \mathrm{~m}$ INT (Isaac Newton Telescope). The log of the observations can be found in Table 1.

All these data were reduced following standard IRAF $^{1}$ procedures.

\subsection{Long-slit spectroscopy}

An $3600 \mathrm{~s}$ spectrum with the slit at parallactic angle $\left(\mathrm{PA}=284^{\circ}\right)$, crossing the centre of the inner nebula, was taken with IDS (Intermediate Dispersion Spectrograph) on the INT on March 9, 2009. The R300V grating was used, centered at $540 \mathrm{~nm}$ and effectively covering from 430 to $810 \mathrm{~nm}$ at a resolving power $R \sim 1500$. The slit width was $1^{\prime \prime}$, while the seeing was $11^{\prime \prime} 8$. HD 192281 was chosen as the standard star to account for flux and sensitivity calibration.

A low-resolution (resolving power $R$ ranging from 290 and 570), 40 min spectrum of M1-75 was taken with ACAM on the WHT on June 11,2009 , with the 400 lines $\mathrm{mm}^{-1}$ transmission VPH (Volume Phase Holographic) disperser, covering the wavelength range between 350 and $950 \mathrm{~nm}$. The $1^{\prime \prime}$ wide slit was positioned at $\mathrm{PA}=0^{\circ}$ in order to get the light from the two central star candidates (see Sect. 4). The seeing was 2!'8, and the standard star was HD 338808.

The spectra were de-biased, flat-fielded, distortion-corrected and wavelength calibrated (from copper-argon and copper-neon arc lamps) using standard IRAF routines. After extraction of the selected nebular and central star features from the orthogonal 2D spectra, the 1D spectra were telluric and sensitivity corrected using the spectrum of the spectrophotometric standard star.

\section{An improved spatio-kinematical model}

The GH $\alpha$ FAS [NII] $658.3 \mathrm{~nm}$ integrated image of M 1-75 is shown in Fig. 2. While no central star (CSPN) is visible in this image, the nebula clearly shows two pairs of lobes with different orientations. They are nested in a central, brighter rim resembling a horseshoe. Both systems of lobes appear distorted and fragmented, and their faint outer edges are difficult to track near the poles.

The lobes of M 1-75 were the subject of a spatio-kinematical model by Dobrinčić et al. (2008). From two slit spectra, approximately along each pair of lobes, a [NII] image from Manchado et al. (1996a), and simple assumptions such as ballistic expansion, they determined the larger and smaller lobes to lie at

1 IRAF is distributed by National Optical Astronomy Observatories. 


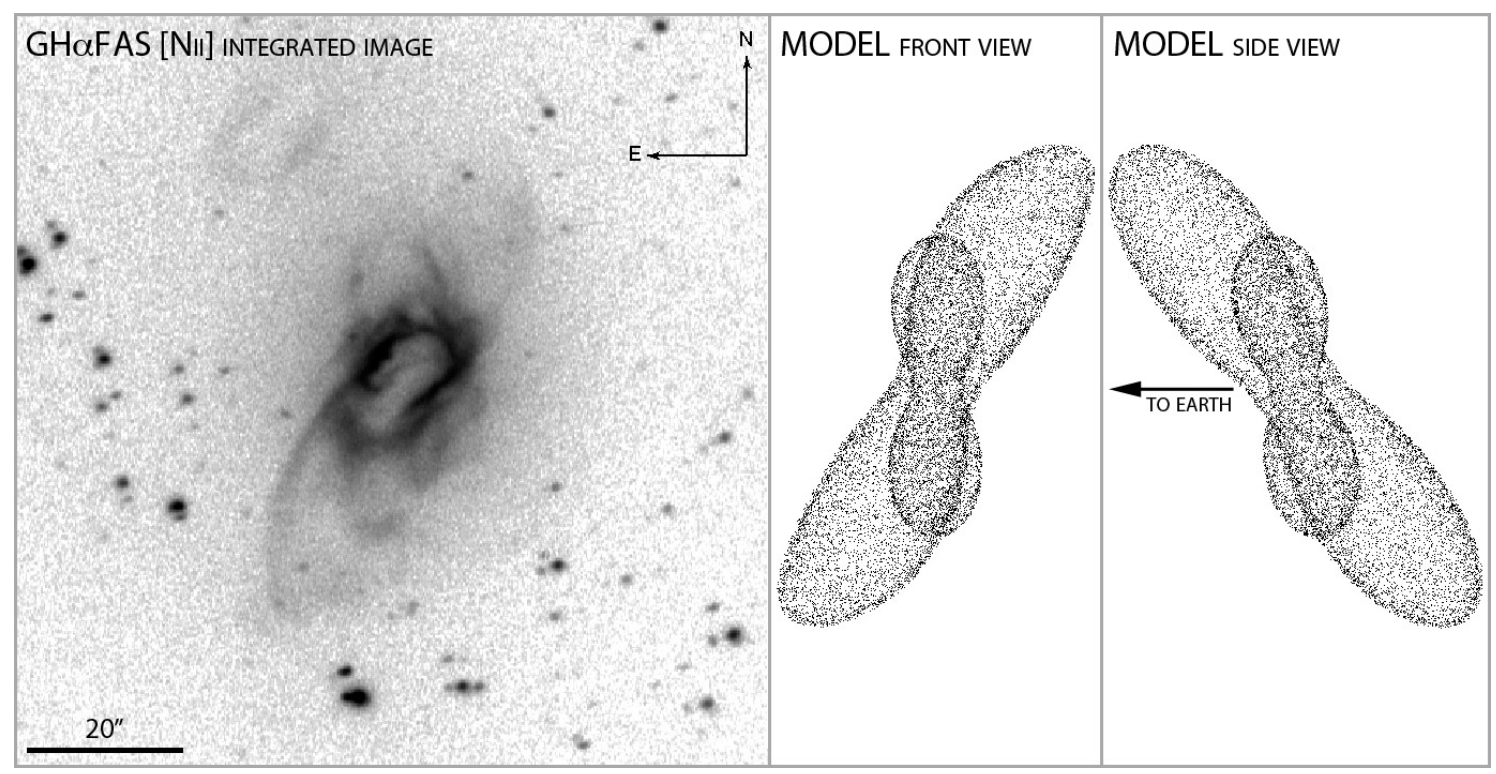

Fig. 2. Left: GHAFAS datacube integrated across every channel to generate a [NII] image of M 1-75. Middle: adopted model of the small and large lobes (see text) as seen on the plane of the sky. Right: transversal view of the adopted model.

inclinations of $87^{\circ}$ and $65^{\circ}$, respectively, and to be likely coeval, with kinematical ages of 2700 and 2400 years per kpc of distance to the nebula, respectively.

Fabry-Perot interferometry (and GH $\alpha \mathrm{FAS}$, in particular) represents a significant step forward in spatio-kinematical modelling of planetary nebulae. Not only does it allows for a resolution in wavelength comparable to high-resolution echelle spectrographs, but also the series of "Doppler-map" images it produces span the whole nebula, instead of being limited by a narrow slit whose orientation has to be decided a priori based on previous images. From the spatio-kinematical point of view, a single $\mathrm{GH} \alpha \mathrm{FAS}$ data cube encompasses everything that is needed (i.e. information of the emission both in the plane of the sky and along the line of sight), its quality being only limited by seeing.

In particular, it is worth noticing the faint, high velocity emission regions offset from the axis of the larger lobes (see Fig. 3), near the polar caps (especially in the southwest region). Those certainly would have remained unnoticed, had we been constrained by a narrow slit oriented along the lobes' "expected" axis.

\subsection{Solf-Ulrich model}

The data cube, with Doppler-shift images spread across 48 channels, allowed us to build a spatio-kinematical model of both systems of lobes (see Santander-García et al. 2004, for a detailed description of the method). Our first approach for each pair of lobes consisted of fitting a Solf-Ulrich (Solf \& Ulrich 1985) surface to the data. This analytical model is described, in spherical coordinates, by:

$r=t D^{-1}\left[v_{\text {equator }}+\left(v_{\text {polar }}-v_{\text {equator }}\right) \sin |\theta|^{\gamma}\right]$

where $r$ is the angular distance to the centre of the nebula (i.e. the adopted central star, see Sect. 4), $t D^{-1}$ the kinematical age of the outflow per unit distance to the nebula, $v_{\text {polar }}$ and $v_{\text {equator }}$ the velocities of the model at the pole and equator respectively, $\theta$ the latitude angle of the model, and $\gamma$ a dimensionless shaping factor. This assumes that each gas particle travels in the radial

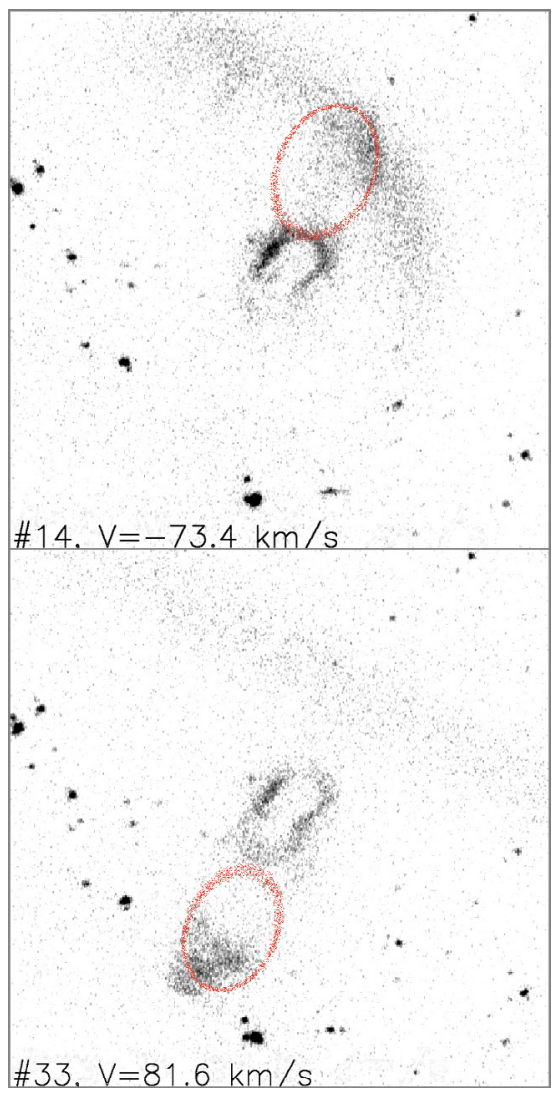

Fig. 3. Two GH $\alpha$ FAS channels with the initial Solf-Ulrich model of the large lobes overimposed (in red). The $V$ label shows the radial velocity (with respect to the centre of the nebula) of the corresponding channels. The size of each frame is $96^{\prime \prime} \times 96^{\prime \prime}$. The emission near the poles (specially the southern one) presents a significant offset from the model symmetry axis.

direction, with a velocity proportional to its distance to the central source (i.e. in a "Hubble-like" flow).

The two-dimensional generatrix is rotated around the symmetry axis and homogeneously populated with particles to 


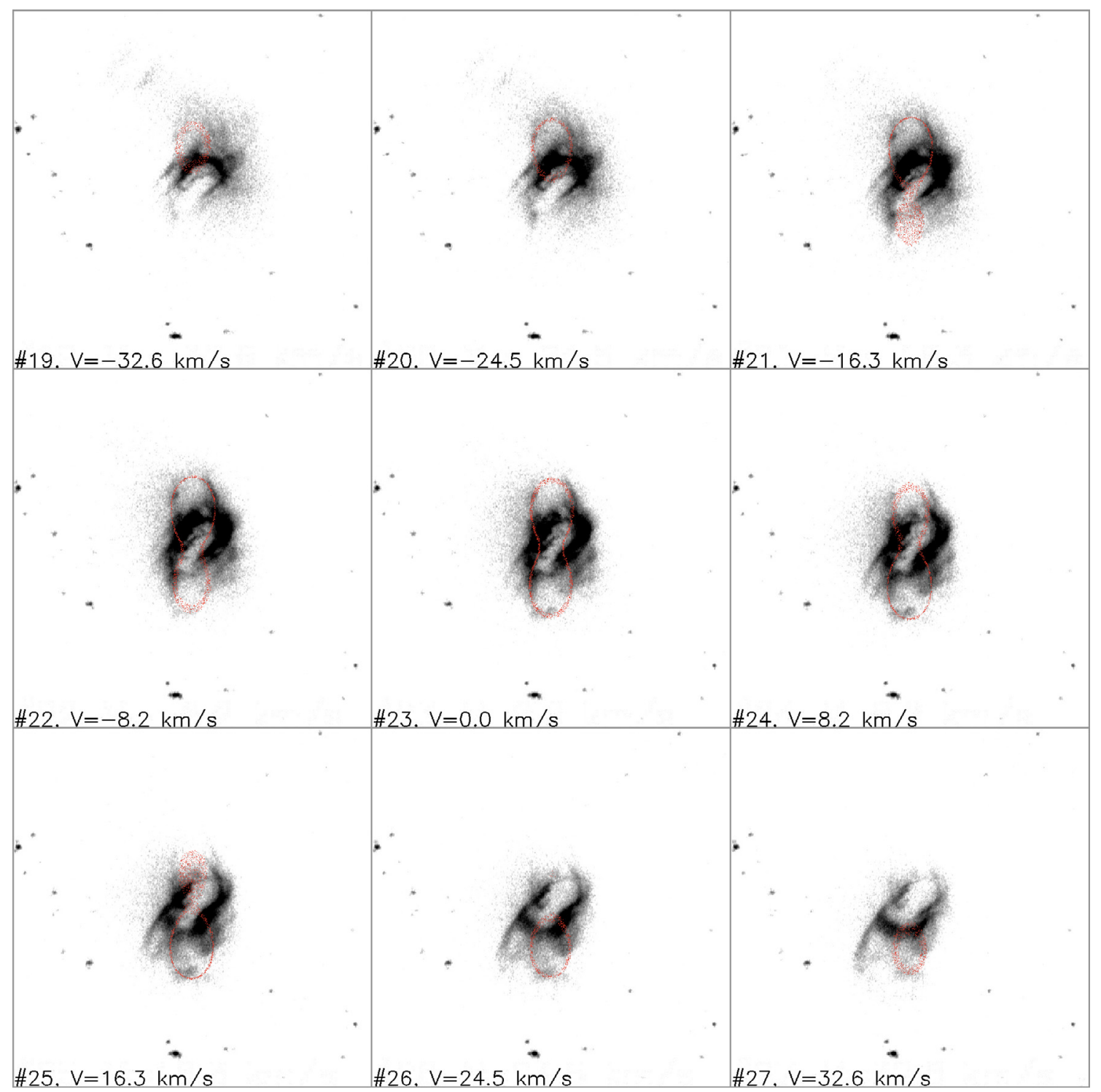

Fig. 4. GH $\alpha$ FAS data of M 1-75 with the Solf-Ulrich model of the small lobes overimposed (in red). The size of each frame is $96^{\prime \prime} \times 96^{\prime \prime}$.

produce a three-dimensional model, and then inclined to the plane of the sky. The resulting geometrical shape and velocity field - once offset by a certain systemic velocity - are then used to generate a simplified image of the nebula and a series (one per GH $\alpha$ FAS channel) of Fabry-Perot synthetic interferometric images for direct comparison with the [NII] integrated image and $\mathrm{GH} \alpha \mathrm{FAS}$ channels data. The irregular surface brightness distribution is beyond the scope of this paper and therefore has not been fitted.

In order to find the best representation of the nebular geometry and expansion, we allow the parameters to vary over a broad range of values and visually compare each resulting model to the integrated image and each of the $48 \mathrm{GH} \alpha \mathrm{FAS}$ channels, until we obtain the best fit. The range of uncertainty is also derived by eye, by individually changing each parameter away from the best fit, until the resulting model is no longer a fair fit to the data. Note that, although the inclination of each pair of lobes cannot be directly determined from the horseshoe - a clearly non-elliptical waist - this fact does not prevent us from finding its value with certain degree of accuracy, given that the aforementioned parameters are disentangled from one another in the results they produce (i.e. there are no degeneracies in the resulting model).
A fair overall fit to the data was obtained for the large and small lobes (Figs. 3 and 4), although the model of the former does not account for the offset emission near the poles. The systemic velocity of both system of lobes was found to be $v_{\text {Sys }}$ LSR $13 \pm 4 \mathrm{~km} \mathrm{~s}^{-1}$. They were also found to share a similar kinematical age of $\sim 1000 \mathrm{yr} \mathrm{kpc}^{-1}$, within uncertainties. The orientations on the sky are instead different - the larger lobes lie inclined $58^{\circ}$ to the line of sight, while the inclination of the smaller pair is $79^{\circ}$. Note that these results (ages, velocities and inclinations) are essentially different from the fit by Dobrinčić et al. (2008), resulting from two slit positions. We were unable to fit a model with their parameters to the GH $\alpha$ FAS data. As their model is based just on two slits rather than the 2D full kinematical information present in the GH $\alpha$ FAS data this is possibly to be expected. The parameters corresponding to our best results for the smaller and larger lobes, together with the uncertainties, are shown in Tables 2 and 3 respectively.

However, no standard Solf-Ulrich model can reproduce the high-velocity emitting region offset from the larger lobes axis. Instead, a modified, point-symmetric Solf-Ulrich model can account for these structures while still fairly fitting the inner regions. 
Table 2. Best-fitting parameters for the small lobes. ":" means uncertain.

\begin{tabular}{ccc}
\hline \hline $\begin{array}{c}\text { Parameter } \\
\text { Small lobes }\end{array}$ & Value & Range \\
\hline$t D^{-1}\left(\mathrm{yr} \mathrm{kpc}^{-1}\right)$ & 925 & $(800-1000)$ \\
$v_{\text {equator }}\left(\mathrm{km} \mathrm{s}^{-1}\right)$ & $15-20$ & $(:)$ \\
$v_{\text {polar }}\left(\mathrm{km} \mathrm{s}^{-1}\right)$ & 105 & $(90-125)$ \\
$\gamma$ & 4.5 & $(4-5)$ \\
$\left.\mathrm{PA}^{\circ}\right)$ & 359 & $(355-1)$ \\
$i\left(^{\circ}\right)$ & 79 & $(76-82)$ \\
$v_{\text {SyS SSR }_{\text {SS }}}$ & 13 & $(11-15)$ \\
\hline
\end{tabular}

\subsection{Point-symmetry, modified Solf-Ulrich model}

In order to find a better fit to the $\mathrm{GH} \alpha \mathrm{FAS}$ data, we introduced the following modified Solf-Ulrich model:

$r=t D^{-1}\left[v_{\text {equator }}+\left(v_{\text {polar }}-v_{\text {equator }}\right) \sin |\theta|^{\gamma(\theta)}\right]$

where $\gamma(\theta)$ is described by

$\gamma(\theta)=\gamma_{\text {equator }}+\left(\gamma_{\text {polar }}-\gamma_{\text {equator }}\right)\left(\frac{2 \theta}{\pi}\right)^{\epsilon}$

where $\gamma_{\text {equator }}$ and $\gamma_{\text {polar }}$ are the values of the shape factor $\gamma$ at the equator and poles respectively, while $\epsilon$ is the power of the dependence (i.e. 1 linear, 2 quadratic, etc.). This dependence of $\gamma$ on the latitude, although increasing the number of free parameters, allows us to better sample the degree of collimation of the nebula at different latitudes.

The next step was adding point-symmetry to the model. We achieved this in a simple way by defining the nebular axes $x, y, z$ ( $x$ along the line of sight towards the viewer, $y$ towards the right, and $z$ upwards along the nebula main axis), and then horizontally projecting the model's $z$ axis on to curves given by

$x^{\prime}=k_{x} z^{p}$

and

$y^{\prime}=k_{y} z^{p}$

where $k_{x}$ and $k_{y}$ are constants, and $p$ is an odd integer (so that it produces a point-symmetric structure). The modified model allows two independent degrees of point symmetry, along the $x$ and $y$ axes, respectively (in a corkscrew fashion). We finally rotated the model by a $\phi$ angle around the $z$ axis before inclining it to the line of sight and produced the synthetic image and $\mathrm{GH} \alpha \mathrm{FAS}$ channel data as described in Sect. 3.1.

Only the large lobes were fit with this model. The best fit values along with their uncertainties - fully consistent with the standard Solf-Ulrich model except for the curvature - are listed in the lower part of Table 3. Almost all the emission from the large lobes, including the aforementioned offset region, was found to be faithfully accounted for by the latter model (see Fig. 5), which added a slight corkscrew-like curvature.

\section{The central star}

The WFC Strömgren Y image (see Fig. 6 top right), where the nebular emission is practically absent, shows two faint stars inside the horseshoe region of the nebula. The star labelled as A is offset $\sim 5^{\prime \prime}$ with respect to star B, which lies approximately at the centre of the nebular emission. In order to gain some insight
Table 3. Top: best-fitting parameters for the large lobes using a standard Solf-Ulrich model. Bottom: best-fitting parameters for the large lobes using a point-symmetric, modified Solf-Ulrich model.

\begin{tabular}{ccc}
\hline \hline Parameter & Value & Range \\
Large lobes & & \\
Solf-Ulrich model & & \\
\hline$t D^{-1}\left(\mathrm{yr} \mathrm{kpc}^{-1}\right)$ & 1000 & $(900-1150)$ \\
$v_{\text {equator }}\left(\mathrm{km} \mathrm{s}^{-1}\right)$ & 25 & $(23-31)$ \\
$v_{\text {polar }}\left(\mathrm{km} \mathrm{s}^{-1}\right)$ & 180 & $(160-200)$ \\
$\gamma$ & 7 & $(6.5-7.5)$ \\
$\mathrm{PA}\left({ }^{\circ}\right)$ & 337 & $(336-339)$ \\
$i\left(^{\circ}\right)$ & 58 & $(54-63)$ \\
$v_{\text {sys } S R}$ & 13 & $(11-15)$ \\
\hline Point-symmetric model & & \\
$t D^{-1}\left(\mathrm{yr} \mathrm{kpc}^{-1}\right)$ & 1000 & $(900-1100)$ \\
$v_{\text {equator }}\left(\mathrm{km} \mathrm{s}^{-1}\right)$ & 25 & $(23-31)$ \\
$v_{\text {polar }}\left(\mathrm{km} \mathrm{s}^{-1}\right)$ & 190 & $(170-210)$ \\
$\gamma_{\text {equator }}$ & 1 & $(1-3)$ \\
$\gamma_{\text {polar }}$ & 14 & $(13-15)$ \\
$\epsilon$ & 0.6 & $(0.55-0.7)$ \\
$\left.\mathrm{PA}^{\circ}\right)$ & 338 & $(337-339)$ \\
$i\left({ }^{\circ}\right)$ & 58 & $(55-62)$ \\
$k_{x}$ & $2 \times 10^{-5}$ & $(1-3) \times 10^{-5}$ \\
$k_{y}$ & $8 \times 10^{-5}$ & $(6-9) \times 10^{-5}$ \\
$p$ & 3 & - \\
$\phi\left(^{\circ}\right)$ & 166 & $(150-185)$ \\
$\left.v_{\text {sys }}\right)$ & 13 & $(11-15)$ \\
\hline
\end{tabular}

on the possibility of either star being the CSPN, we took multicolour ( $U, B, V$ and $I$ ) WFC images of the nebula (see Fig. 6) and an ACAM 40 min low-resolution spectrum of both star candidates. Unfortunately, the spectrum of each star only shows the nebular emission lines together with a continuum whose signal to noise is too low $(\sim 10-15)$ to allow us to detect any photospheric spectral signatures of a white dwarf. Instead, once the nebular emission has been accounted for, we can estimate the visual magnitudes of both stars. This results in $m_{\mathrm{v}} \sim 19.3$ for star A and $m_{\mathrm{v}} \sim 21.4$ for star B.

On the other hand, star A is barely visible in the images in the light of the $U$ and $B$ bands, and clearly visible in the $V$ and $I$ bands, while star B is only visible in the latter bands. Although those bands are highly contaminated by strong emission from the nebula, we were able to roughly estimate the $V-I$ colour of star B. In order to do this, we added 7 rows $(\sim 1.5 \times F W H M)$ centred on the star and with $\mathrm{PA}=138^{\circ}$, where the nebular contamination is minimum. For each image, a gaussian was fitted to the star profile, taking a linear fit between the base of the wings of the profile as background. Once the quantum efficiency of the detector (EEV 4280), the filter transmissions and the atmospheric extinction have been taken into account, we found an observed $V-I \sim 2.0$ for star B.

The dereddening of this value is not straightforward, due to the extinction variation across the nebula. The extinction values found in the literature range from $c_{\mathrm{b}}=2.29$ to $c_{\mathrm{b}}=2.9$ (Hua 1988; Bohigas 2001) from the Balmer decrement for different regions of the nebula. From our own ACAM and IDS spectra, we computed an extinction value $c_{\mathrm{b}}=1.9 \pm 0.1$ at the location of star B, close to the values found by other authors. If we assume the star to lie within the nebula, we can deredden its $V-I$ colour using the Fitzpatrick (2004) extinction law, assuming $R=3.1$, to obtain an intrinsic $(V-I)_{0} \sim 0$ for star B. 


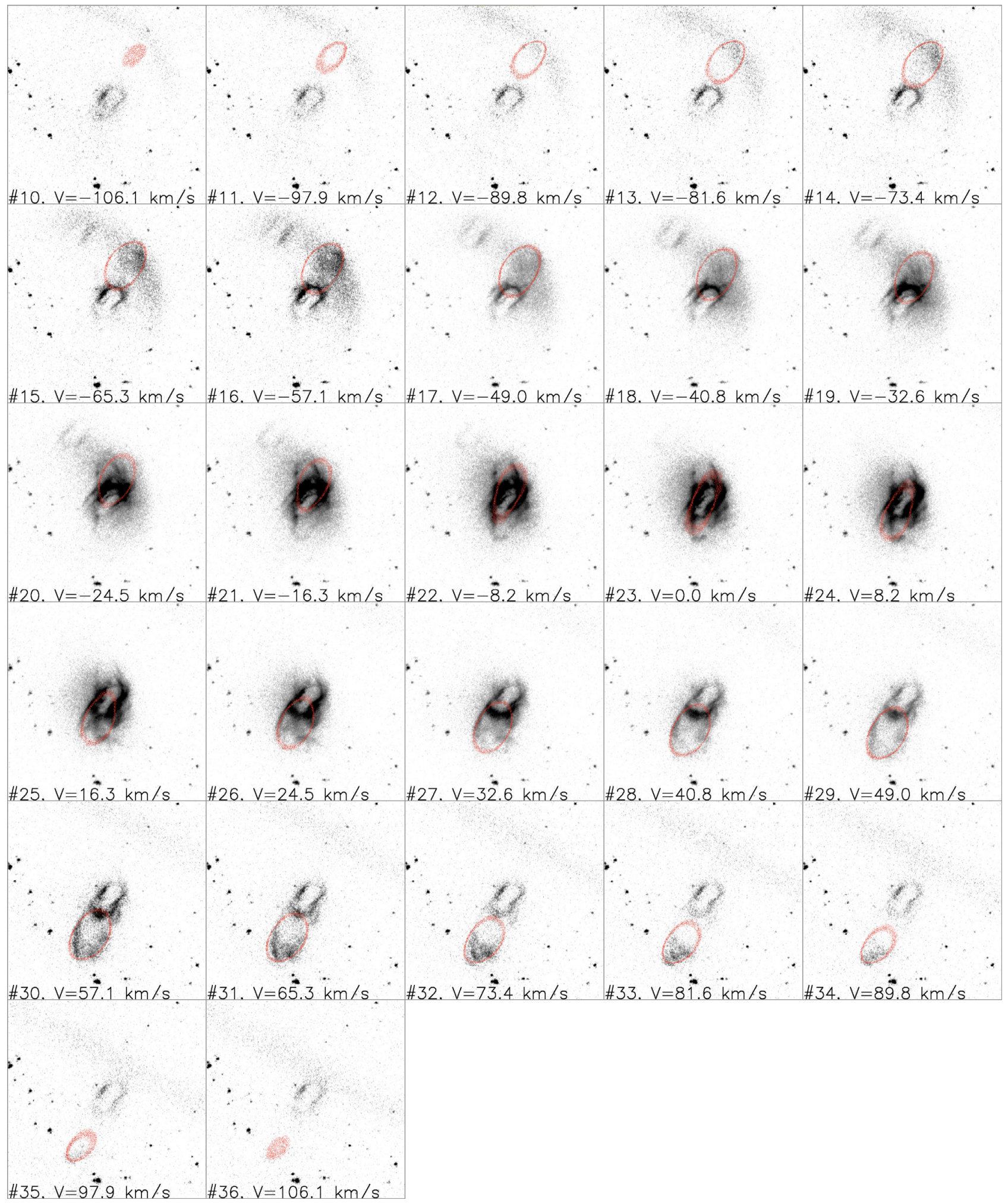

Fig. 5. GH $\alpha$ FAS data of M 1-75 with the modified Solf-Ulrich, point-symmetric model of the large lobes overimposed (in red). The size of each frame is $96^{\prime \prime} \times 96^{\prime \prime}$. The brightness/contrast of channels \#17 to \#29 have been modified to provide a better display of the central region, although the noise from the background has also been amplified as a result. The horseshoe ghost is clearly visible near the top left corner in several channels, while the arc-shaped artifact can be seen progressively crossing the frame towards its centre.

\section{Discussion}

\subsection{The shaping of the nebula}

Our spatio-kinematical modelling confirms the presence of two pairs of nested lobes in the nebula of M 1-75, although with essentially different velocities and ages from those found by Dobrinčić et al. (2008). For reasons outlined in Sect. 3, we consider our results more reliable. The outer lobes show some evidence of departure from axial symmetry in the polar regions, which we have modelled by applying a slight degree of 


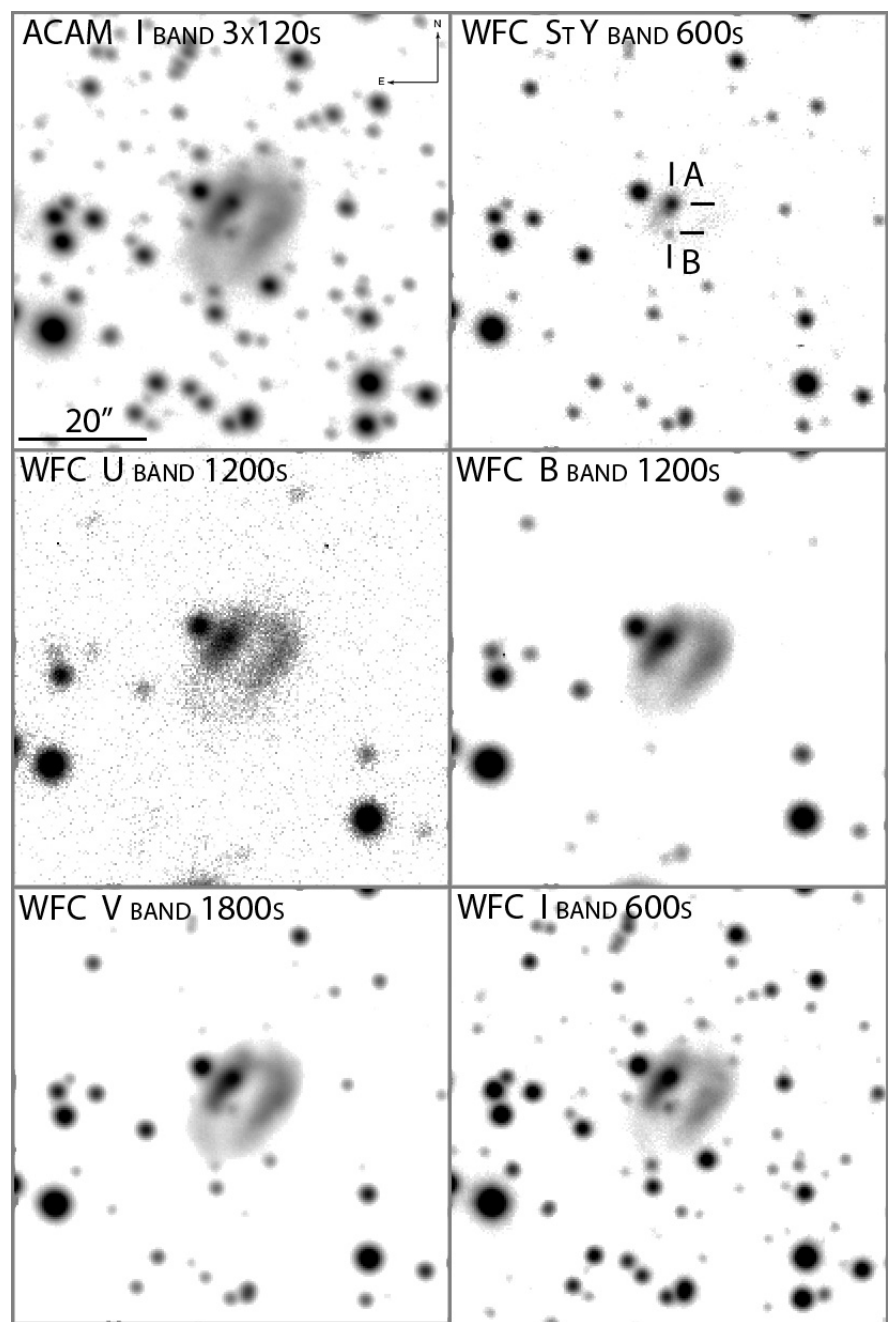

Fig. 6. Multiband images of M 1-75. Each frame is labelled according to the instrument, band and exposure time. Stars A and B (see text) are labelled in the Strömgren Y image, where the emission from the nebula is practically absent
Given all the aforementioned, it is not trivial to depict a formation scenario for M 1-75. The classic Generalised Interacting Stellar Winds model (GISW, Balick et al. 1987; a refined version of the original ISW by Kwok et al. 1978) model. In this model, the isotropic, fast and tenuous wind from a post asymptotic giant branch (AGB) star interacts with the anisotropic, slow and dense winds previously deployed by the star during the AGB stage and shapes a bipolar nebula, is not sufficient to explain either the presence of a multipolar structure, or the slight degree of pointsymmetry of the larger lobes. Instead, one has to invoke a mechanism such as the warped-disc proposed by Icke (2003): if the post-AGB is surrounded by a disc, warped by radiative instabilities, the wind interaction could result in a multipolar nebula with some degree of point-symmetry in the external regions (e.g. NGC 6537). The origin of the disc itself (i.e. the necessary equatorial density enhancement), however, would still require either the CSPN to be actually a close binary, or to have engulfed one of its planets as it died. A more complex approach is that of Blackman et al. (2001), in which a low-mass companion originates a disc blowing its own wind. A misalignment between the stellar and disc magnetic and rotational axes could give rise to a quadrupolar structure, while the point-symmetry observed in the tips of the larger lobes would require precession of the magnetic axes. We consider the model proposed by Manchado et al. (1996b), in which a fast precessing disc is responsible for the different orientation of each structure, as a less likely scenario, for it would require both structures to have been ejected in extremely rapid succession (in $\sim 300-500 \mathrm{yr}$ to still fit our spatiokinematical model uncertainties, considering the distance range adopted below and the spatial angular separation of the inner and outer lobes), and it would not explain the point-symmetryic structure.

It is noteworthy that all these models require the CSPN to be/have been a close-binary system (or at least a single star with a close massive hot Jupiter) for the disc to have formed. There is, to our knowledge, no plausible model in the extensive literature able to produce a quadrupolar (not to mention point-symmetric) $\mathrm{PN}$ out of a single star.

\subsection{The central star}

Although it constitutes the cornerstone for many parameters of $\mathrm{PNe}$ and their central stars, such as the kinematical age of the nebula or their total luminosity, the distance to these objects is poorly known in most cases. M 1-75 is no exception. In the literature one can find several distances based on different methods, such as the statistical distance range, 2.6-3.7 kpc by Cahn et al. (1992), or the Galactic rotation curve distance of $5.3 \mathrm{kpc}$ by Burton (1974). The more recent extinction-distance method of Sale et al. (2009), easily applicable to the INT Photometric $\mathrm{H} \alpha$ Survey (IPHAS) data sample and reliable in most $\mathrm{PNe}$ (Giammanco et al., in prep.), does not help in the case of nebulae with a significant amount of internal extinction. In the case of M 1-75, the extinction value lies far above the plateau of the field stars in the extinction-distance graph, confirming a significant internal extinction in this nebula (another possibility would be that stellar $\mathrm{H} \alpha$, possibly from a cooler companion, scatters from dust in or near the inner horseshoe, thereby increasing the $\mathrm{H} \alpha / \mathrm{H} \beta$ ratio; this is ruled out, however, by this ratio in the core being lower than in the horseshoe). Given the lack of evidence favouring a particular distance estimate, rather than adopting a specific distance we will consider a more conservative, intermediate distance range between 3.5 and $5.0 \mathrm{kpc}$. 
Probably due to its internal extinction, so far there has been no clear evidence of the CSPN of M 1-75, other than a slight enhancement of the isophotes in an [O III] image (Hua 1988). Our images in the light of Strömgren Y, followed by low resolution spectra, have detected two candidates to CSPN (Fig. 6), stars A and B (at the position pointed out by Hua), of apparent magnitudes $m_{v} \sim 19.3$ and 21.4 respectively. Unfortunately, the low signal to noise ratio in a 40 min spectrum with ACAM ( 15 in the continuum of the brightest star around $700 \mathrm{~nm}$ ) prevents us from detecting and analysing any photospheric features, leading us to think that any future research on these stars will require an 8-m class telescope.

Although several nebulae have offset CSPN, it is unlikely that star A is the central star of M 1-75. Even in the most extreme case known (MyCn 18; Sahai et al. 1999), the star is nowhere in contact with the equatorial waist of its nebula. In fact, to explain such an offset $\left(\sim 5^{\prime \prime}\right)$, one would need to invoke a high proper motion central star travelling $\sim 10-20 \mathrm{~km} \mathrm{~s}^{-1}$ faster than its own nebula since the ejection, 3500-5000 yr ago at the adopted distance range. The nebula would have to have been heavily braked by interaction with the ISM, being distorted in the process. However, making a simple extrapolation from the PN-ISM interaction models for a round nebula by Wareing et al. (2007), every symmetry in the system would have been long lost at such a late stage of interaction.

Therefore we can safely rule out star A and assume star B, at the centre of the nebula, as the CSPN of M 1-75. The visual magnitude we have derived for this star is consistent with the estimate by Hua (1988) who, assuming a distance of $2.8 \mathrm{kpc}$ (Acker 1978), suggested a hot $\left(\log T_{\text {eff }}=5.3\right)$ core with a mass of about $0.57-0.6 M_{\odot}$ and a luminosity of $\log L / L_{\odot}=2.36$. The kinematical age of the nebula found in this work is coherent with the luminosity and $T_{\text {eff }}$ of the fading evolutionary track of a hydrogen-burning high-mass $\left(\sim 0.6-0.8 M_{\odot}\right)$ core (Schönberner 1993; Mendez \& Soffner 1997).

Based on the extremely high $\mathrm{N} / \mathrm{O}=2.85$ and $\mathrm{He} / \mathrm{H}=0.18$ of the nebula, Guerrero et al. (1995) hinted towards the possibility of the CSPN actually being a post-common envelope close binary with a total initial mass between 4 and $6 M_{\odot}$. In fact, the $(V-I)_{0} \sim 0$ colour estimated in this work is considerably redder than the value of $V-I=-0.9$ one would expect from a single blackbody of $\log T_{\text {eff }}=5.3$. This might suggest the presence of a fainter $\left(L_{\text {bol }}<10^{-3} \times L_{\text {bolwD }}\right)$, much colder $\left(T_{\text {eff }} \lesssim 10000 \mathrm{~K}\right)$ companion star, as together they would produce $V-I$ and luminosities coherent with the observations. This would be consistent with the increasing number of confirmed binary cores hosting bipolar PNe (Miszalski et al. 2009; Miszalski et al., in prep.), but would need to be proved via a direct method (e.g. photometric monitoring).

\section{Summary and conclusions}

A spatio-kinematical model of the M 1-75 nebula has been presented. Two pairs of lobes emerge from the core, their expansion patterns well described by a Hubble-like flow, their kinematical ages $\left(\sim 1000 \mathrm{yr} \mathrm{kpc}^{-1}\right)$ being similar within uncertainties, while their orientations differ by $\sim 22^{\circ}$. The outer lobes have been found to be slightly point-symmetric. The implications of these results on the different formation theories have been briefly discussed, and a model invoking a close companion star (or a hot Jupiter planet) has been favoured.

On the other hand, the $V-I$ colour and brightness of the CSPN - first identified in this work - are compatible with the presence of a close companion provided its $T_{\text {eff }}$ is less than $10000 \mathrm{~K}$ and its luminosity less than $10^{-3}$ times that of the white dwarf.

Acknowledgements. M.S.G. would like to thank Mariano Santander for his help with the point-symmetric model, and Guillermo García-Segura for his insight on single stars and quadrupolar nebulae.

\section{References}

Acker, A. 1978, A\&A, 33, 367

Balick, B., \& Frank, A. 2002, ARA\&A, 40, 439

Balick, B., Preston, H. L., \& Icke, V. 1987, AJ, 94, 1641

Blackman, E. G., Frank, A., \& Welch, C. 2001, ApJ, 546, 288 Bohigas, J. 2001, RMxAA, 37, 237

Burton, W. B. 1974, Galactic and Extra-Galactic Radio Astronomy, 82

Cahn, J. H., Kaler, J. B., \& Stanghellini, L. 1992, A\&AS, 94, 399

de Marco, O. 2009, PASP, 121, 316

Dobrinčić, M., Villaver, E., Guerrero, M. A., \& Manchado, A. 2008, AJ, 135, 2199

Fitzpatrick, E. L. 2004, in Astrophysics of Dust, ed. A. N. Witt, G. C. Clayton, \& B. T. Draine, ASP Conf. Ser., 309, 33

García-Segura, G., Langer, N., Różyczka, M., \& Franco, J. 1999, ApJ, 517, 767

Guerrero, M. A., Stranghellini, L., \& Manchado, A. 1995, ApJ, 444, L49

Hernandez, O., Fathi, K., Carignan, C., et al. 2008, PASP, 120, 665

Hua, C. T. 1988, A\&A, 193, 273

Icke, V. 2003, A\&A, 405, L11

Kwok, S., Purton, C. R., \& Fitzgerald, P. M. 1978, ApJ, 219, L125

Manchado, A., Guerrero, M. A., Stanghellini, L., \& Serra-Ricart, M. 1996a, The IAC morphological catalog of northern Galactic planetary nebulae

Manchado, A., Stanghellini, L., \& Guerrero, M. A. 1996b, ApJ, 466, L95

Mendez, R. H., \& Soffner, T. 1997, A\&A, 321, 898

Miszalski, B., Acker, A., Moffat, A. F. J., Parker, Q. A., \& Udalski, A. 2009, A\&A, 496, 813

Nordhaus, J., \& Blackman, E. G. 2006, MNRAS, 370, 2004

Riera, A. 1990, Ph.D. Thesis, Universidad de la Laguna

Sahai, R., Dayal, A., Watson, A. M., et al. 1999, AJ, 118, 468

Sale, S. E., Drew, J., Unruh, Y. C., et al. 2009, MNRAS, 392, 497

Santander-García, M., Corradi, R. L. M., Balick, B., \& Mampaso, A. 2004, A\&A, 426, 185

Schönberner, D. 1993, in Planetary nebulae, ed. R. Weinberger, \& A. Acker (Dordrecht: Kluwer Academic), IAU Symp., 155, 415

Solf, J., \& Ulrich, H. 1985, A\&A, 148, 274

Wareing, C., Zijlstra, A. A., \& O’Brien, T. J. 2007, MNRAS, 382, 1233 